\title{
Which Physiological Components are More Suitable for Visual ERP Based Brain-Computer Interface? A Preliminary MEG/EEG Study
}

\author{
Luigi Bianchi $\cdot$ Saber Sami $\cdot$ Arjan Hillebrand · \\ Ian P. Fawcett · Lucia Rita Quitadamo • \\ Stefano Seri
}

Received: 23 July 2009/Accepted: 2 April 2010/Published online: 20 April 2010

(C) Springer Science+Business Media, LLC 2010

\begin{abstract}
We investigated which evoked response component occurring in the first $800 \mathrm{~ms}$ after stimulus presentation was most suitable to be used in a classical P300-based brain-computer interface speller protocol. Data was acquired from 275 Magnetoencephalographic sensors in two subjects and from 61 Electroencephalographic sensors in four. To better characterize the evoked physiological responses and minimize the effect of response overlap, a $1000 \mathrm{~ms}$ Inter Stimulus Interval was preferred to the short $(<400 \mathrm{~ms})$ trial length traditionally used in this class of BCIs. To investigate which scalp regions conveyed information suitable for BCI, a stepwise linear discriminant analysis classifier was used. The method iteratively analyzed
\end{abstract}

This is one of several papers published together in Brain Topography on the "Special Topic: Cortical Network Analysis with EEG/MEG'.

L. Bianchi $(\bowtie) \cdot$ L. R. Quitadamo

Department of Neuroscience, University of Rome

"Tor Vergata", Via Montpellier 1, 00135 Rome, Italy

e-mail: luigi.bianchi@uniroma2.it

L. Bianchi · L. R. Quitadamo

Laboratory of Neurophysiopatology, S. Lucia Foundation,

Rome, Italy

L. Bianchi · L. R. Quitadamo

Centro Di Biomedicina Spaziale, University of Rome

"Tor Vergata", Rome, Italy

S. Sami · I. P. Fawcett · S. Seri

The Wellcome Trust Laboratory for MEG Studies, School of

Life and Health Sciences, Aston University, Birmingham, UK

A. Hillebrand

Department of Clinical Neurophysiology, VU University

Medical Center, De Boelelaan, 1118, 1081 HV Amsterdam,

The Netherlands each individual sensor and determined its performance indicators. These were then plotted on a 2-D topographic head map. Preliminary results for both EEG and MEG data suggest that components other than the P300 maximally represented in the occipital region, could be successfully used to improve classification accuracy and finally drive this class of BCIs.

Keywords Brain-computer interface - P300 - MEG · EEG $\cdot$ SWLDA

\section{Introduction}

Brain-computer interface (BCI) systems represent a means of communication for individuals who experience impairment in expressing their intents to the external world and so a way to reduce the impact of disability caused by diseases such as multiple sclerosis, trauma, stroke (Wolpaw et al. 2002). A BCI translates brain signals measured from the subject into features suitable for driving an external device such as a speller, a wheelchair, or a robotic arm.

A visual P300-based BCI exploits the P300 response, which can be recorded on the electroencephalogram (EEG) in response to rarely occurring events embedded in a set of frequent events with different physical properties. These events are usually represented by tone pitches or images. If attention is focused on the rare event, a time and phaselocked response of positive polarity-the $\mathrm{P} 300$-is evoked in the EEG approximately $300 \mathrm{~ms}$ after its presentation. By detecting the events responsible for the P300, we can infer the intent that the subject wishes to communicate.

One of the most frequently used P300-based BCI system relies on a speller represented by a matrix of symbols displayed on a computer screen (Donchin et al. 2000). By 
focussing on specific elements of the matrix, the user can achieve a simple but effective communication. Optimization of this kind of BCI systems has received increasing attention in recent years (Sellers et al. 2006; Serby et al. 2005; Nijboer et al. 2008; Allison and Pineda 2006; Krusienski et al. 2008). In these studies the inter stimulus interval (ISI) is usually set in the $175-350 \mathrm{~ms}$ range, which implies that a stimulation occurs before the previous response has extinguished. Whilst this choice has obvious communication speed advantages, it might hide some aspects related to the physiological response. A recent study has also addressed the issue of the "best electrodes set" to be used, analyzing a limited number of sensor configurations (Krusienski et al. 2008). In this study, we used a classical P300-evoking speller to investigate whether any evoked component, besides the P300, could be used to improve the classification accuracy and, more in general, to evaluate its topographic distribution. We used two electrophysiological modalities-EEG and magnetoencephalography (MEG)-to investigate the potential advantage of the higher spatial resolution and signal to noise ratio of MEG with respect to the traditionally used EEG.

\section{Material and Methods}

Six subjects participated in the study and two different recording techniques were used: EEG (four subjects, three female, one male, mean $\pm \mathrm{SD}=$ age $30.25 \pm 10.04$ ) and MEG (2 male subjects aged 51, 42). MEG was chosen for this BCI protocol because of its higher spatial resolution (Mellinger et al. 2007), offering more precise information about the brain areas involved in the discrimination task, whilst EEG retains a competitive advantage in terms of cost and accessibility. We used a widely applied P300 protocol in BCI research (Donchin et al. 2000) based on a speller constituted by a square matrix of 36 symbols.

In line with the majority of visual P300 protocols, subjects were asked to fixate a specific letter and count the number of times it flashed. Each row and column flashed 15 times with a $1000 \mathrm{~ms}$ inter-stimulus interval with a $100 \mathrm{~ms}$ duration. We chose a longer ISI with respect to previous studies in order to minimize responses overlap.

The rows and columns which do not contain the selected character were defined as non-targets. Twelve different classes of responses-one for each row and column-were separately averaged off-line. A classifier was employed to indentify the two target responses (one row, one column). The attended character was identified as the one located at the intersection of the target row and the target column.

In Fig. 1 a short stimulation segment in which the requested letter is placed in the fourth column is reported. The corresponding sequence of target and non-target stimuli is shown just below the time axis.

In the EEG experiment, each subject was presented with all the 36 letters, for a total of 6,480 stimuli (1,080 targets, 5,400 non-targets), while for the MEG subjects had to concentrate on 18 letters for a total of 3,240 stimuli (540 targets, 2,700 non-targets) each.

In both cases, data were converted to the NPX file format (Bianchi et al. 2007) and then processed with the NPXLab Suite (available at http://www.brainterface.com).

\section{Data Acquisition}

MEG was recorded in a magnetically shielded room using a system (VSM Medtech Ltd, Port Coquitlam, BC, Canada) comprising 275 gradiometers. Data was sampled at $600 \mathrm{~Hz}$ simultaneously with trigger signals generated by specifically designed stimulation software running on a separate PC.
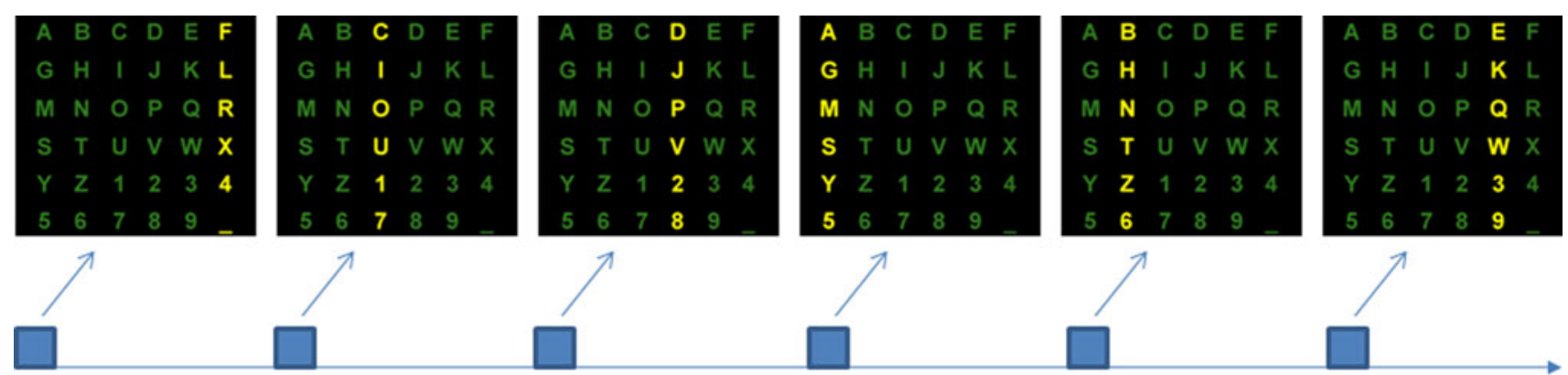

Non TARget
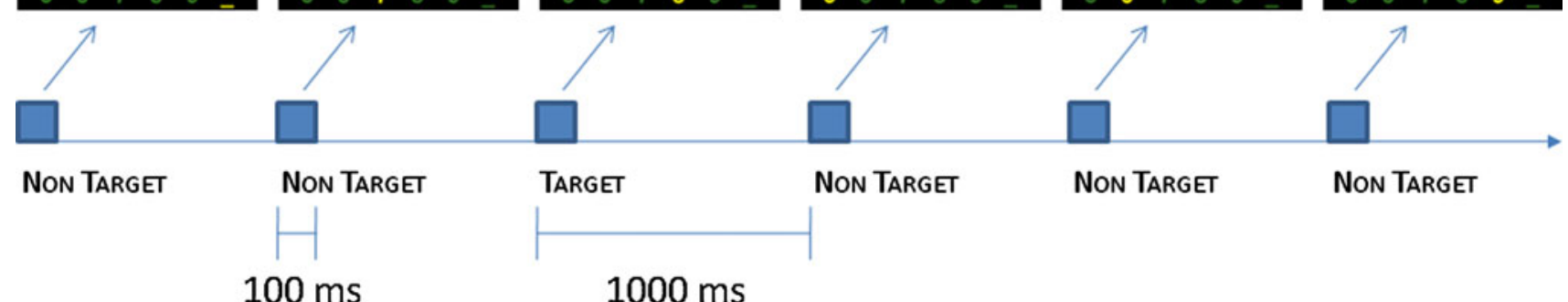

NON TARget

Non TARget

Non TARget

Fig. 1 An example of stimulating sequence (by columns). Every $1000 \mathrm{~ms}$ all the letters of one of the six columns turn from green to light yellow for about $100 \mathrm{~ms}$. This evokes a physiological response if the subject is watching an intensified letter 
EEG was recorded in a silent room using an EBNeuro Mizar system (Florence, Italy) from 61 sensors, with a sampling rate of $256 \mathrm{~Hz}$. Reference electrode was positioned between $\mathrm{AFz}$ and $\mathrm{Fz}$ while ground was between $\mathrm{Pz}$ and $\mathrm{POz}$.

\section{Data Processing}

Acquired data was band-pass filtered (0.5-30.0 Hz). MEG signals were downsampled to $150 \mathrm{~Hz}$.

A stepwise linear discriminant analysis (SWLDA) classifier (Krusienski et al. 2008) was used. It was implemented using the $\mathrm{C}++$ programming language and included in the Body Language Framework (Bianchi et al. 2003) $(\mathrm{BF}++)$. The NPXLab suite, a main component of the $\mathrm{BF}++$, was then used for the evaluation of the performances of the classifier. A screenshot of the classifier option form is shown in Fig. 2.

The classifier was fed with data relative to the first $800 \mathrm{~ms}$ of the signal after the visual stimulations for each sensor, iterated over the whole sensors set. This allowed preservation of topographic information regarding the ability of the classifier to recognize the correct targets.

For all of the six subjects the classifier was trained by using nine of the asked alphanumeric characters, while the classification was performed on the remaining ones. This procedure was automatically iterated, each time randomly choosing a new set of training characters and using the remaining ones for the testing, until 1,080 classifications per sensor were performed.

For each sensor, the amounts of correct, wrong and uncertain (the classifier abstained from decision) classifications were computed and the percentage of the correct ones was represented on a 2-D topographic map using a quadratic interpolation on the eight nearest neighbors to avoid any spatial filtering effect at sensors locations.

\section{Results}

Maps relative to the percentage of correct classifications and relative to the four EEG and the two MEG subjects, respectively are reported in Figs. 3 and 4. Values below $50 \%$ are reported in gray (chance level was $16.66 \%$ ) otherwise they are mapped through a color scale, ranging from red, corresponding to the threshold value, to blue, corresponding to the perfect $(100 \%)$ accuracy. White dots represent sensors positions. From the EEG maps (Fig. 3) it can be easily seen that, except for Subject 1, who confessed lack of attention for all the duration of the protocol, a wide brain area covering either the central or the occipital region provides accuracies above the threshold. The best sensor locations were respectively $\mathrm{FCz}$ (with an accuracy of $52.4 \%)$, PO7 (70.3\%), PO3 (68.3\%) and P6 (92.2\%).

The corresponding maps relative to MEG data are reported in Fig. 4. In this case the brain areas that provide
Fig. 2 The main classifiers form. One can: (1) select the sensors to use (left list), (2) set the segmentation parameters, (3) select the triggering events to use, (4) select the asked characters to use, (5) select the encoder, from which target and non-target triggers are automatically computed. The Classifiers group box (6) shows the actually selected classifiers and allows configuring them. (7) Validation can then be manual or automated (folded)

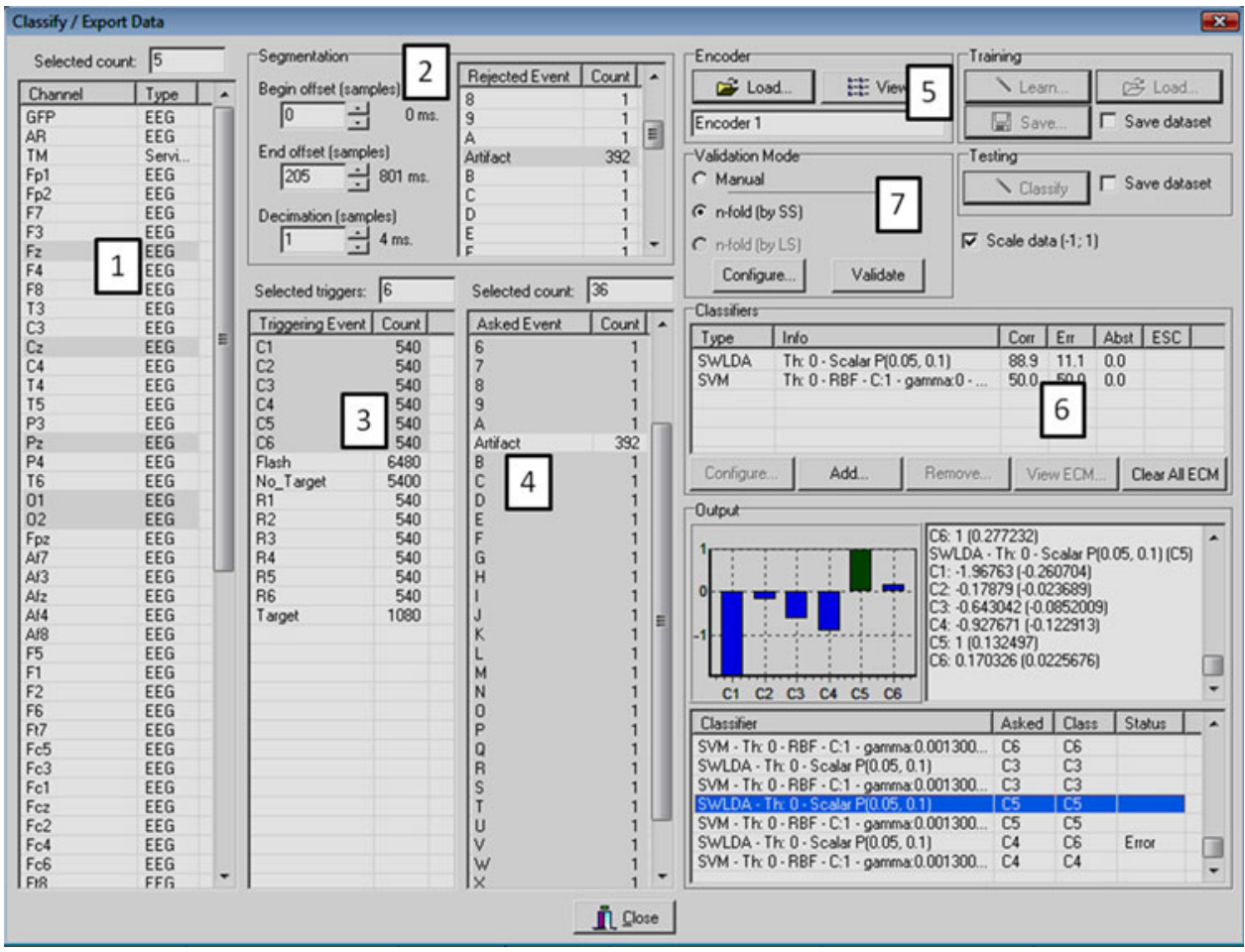




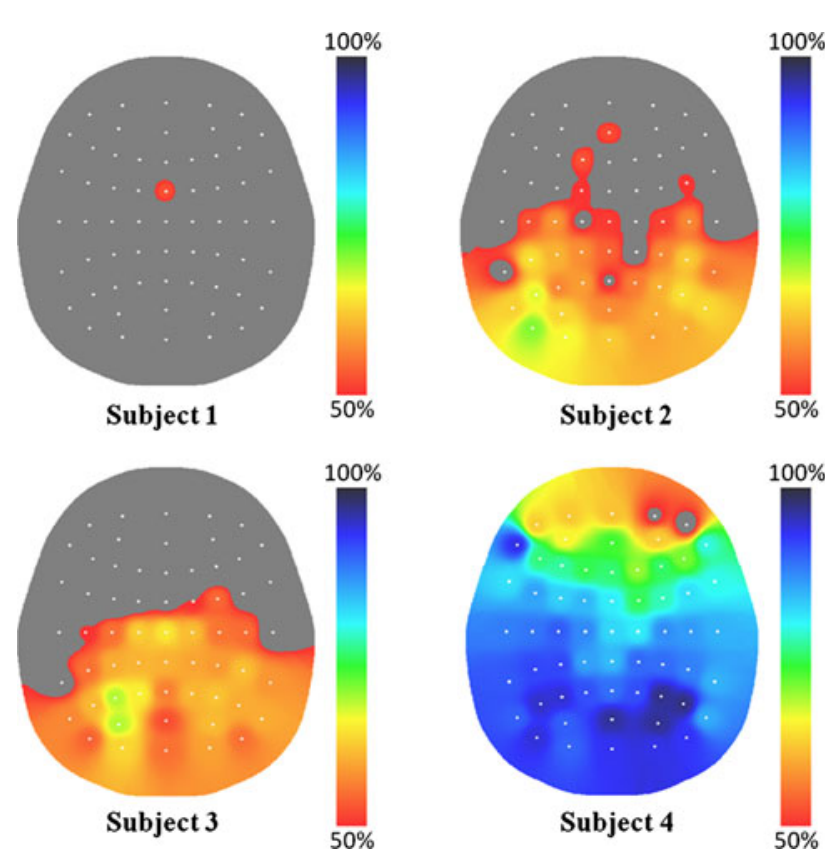

Fig. 3 Subjects 1-4, EEG single sensors classification maps. Values are expressed in percentage

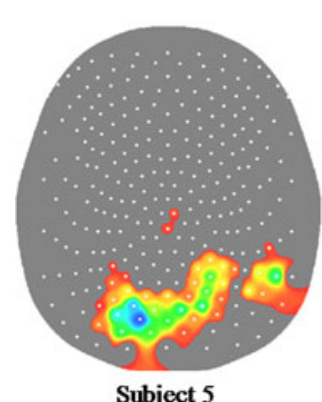

Subject 5

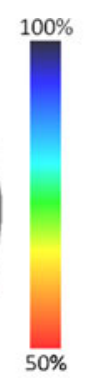

$50 \%$

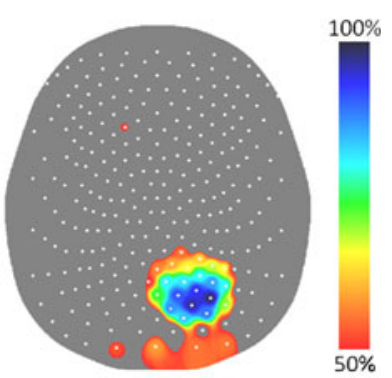

Subject 6
Fig. 4 Subjects 5-6, MEG single sensors classification maps. Values are expressed in percentage

the best accuracies are more focused and localized in the parieto-occipital region. The best performance (90.4\%) was obtained at a left occipital sensors, location MLO31for subject 5 and $98.33 \%$ at location MRO21for subject 6 .

In addition, in order to quantify and point out the different contributes due to the visual N100 and the cognitive P300 components, for subject 4 classifications were performed both with the $0-250 \mathrm{~ms}$ interval after the stimulations, in which the N100 is most prominent, and with the 250-500 ms interval, in which is the P300 to be the most prominent. Subject 4 was chosen because he showed an high activation of the occipital zone, with the classification maps in the 0-800 ms interval (Fig. 3), and an ample N100 component from an event related potentials (ERP) analysis. In Fig. 5 averages of the target (blue lines) and non-target (orange lines) trials over all the letters and computed for each electrode are shown. It is evident that, in the
0-250 ms interval, the N100 component is very predominant, with respect to the $\mathrm{P} 300$ component that is less ample in the 250-500 ms. The other subjects show a similar behavior, even if the P300 component may be larger. In any case, the N100 component is still present and clearly visible.

Finally, results of the classifications with the two intervals are shown in Fig. 6: it is evident that, classifying with the $0-250 \mathrm{~ms}$ interval, almost all the occipital electrodes show the highest accuracy $(100 \%)$, while with the 250-500 ms interval, central electrodes activate the most, even if with lower accuracy $(50-70 \%)$ with respect to the previous case. This confirms that earlier components in the occipital region play an important role in the classification process.

\section{Discussion and Conclusions}

In this study a classical BCI speller protocol has been used to investigate the existence of other physiological components besides the P300 that could be used to increase the classification accuracy and to determine the brain areas in which they are located. In order to keep the responses to the stimulations separate, so avoiding destructive interferences among different overlapping evoked components, a longer ISI than the most used ones, was preferred in the experimental design.

Our preliminary results suggest that the sensors belonging to the occipital region of the brain should be included in the sensors set for this class of protocols. This finding is of particular importance given the rising interest in using only a limited set of sensors in BCIs for practical reasons. It should be mentioned that in one subject (subject 1) the classification accuracy was very poor. The same subject confessed later that had a certain difficulty to stay well concentrated and this can be the main reason. Moreover, it is well known that differences among subjects are usually observed in whatever BCI protocol so that the final performances of these systems might vary from user to user.

The topographic approach used in this experimentusing one sensor at a time for the classification and evaluating the classifier accuracy globally-is a promising data-reduction tool in identifying the brain regions in which the difference between target and non-target responses is maximal. Whilst simultaneous use of multiple sensors is known to provide best results for BCIs, the approach used in the present study allows the preservation of topographic information regarding the ability of the classifier to recognize the correct targets. This approach can be also useful in investigating the role of different brain areas in the discrimination task (target-non-target) typical of $\mathrm{P} 300$-based $\mathrm{BCI}$ protocols. In fact, mapping the 
Fig. 5 Average ERP for Subject 4: orange lines represent the target averages on all the electrodes (one line per electrode), while blue lines represent the corresponding non-target averages. The averages were computed using all of the letters
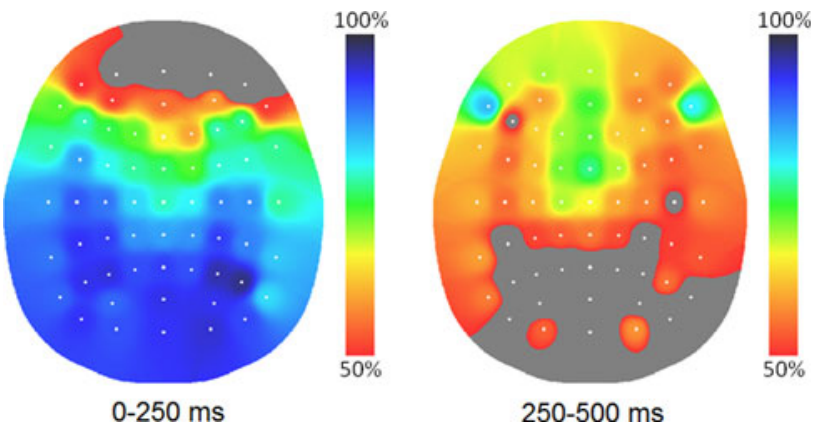

Fig. 6 Classification maps for Subject 4, in the 0-250 ms (left) and 250-500 ms (right) time intervals

classification results could provide us with an indirect measure of brain processing load in the execution of this experimental protocol. In particular, given that the SWLDA classifier tries to maximize differences among a discrete set of features to discriminate classes of data, it will perform better whenever the differences are intrinsically high. One can then deduce that the better the performances of a classifier, the higher the differences among the classes and then of the physiological signals.

The involvement of the parieto-occipital region, as outlined in this experiment by both EEG and MEG data, also suggests that some visual evoked component (e.g. N100) should be used for BCIs in addition to the cognitive ones (P300) in order to improve the performances of current BCIs. This could be done by including specific sensors and/or early components of the physiological responses.

Finally, MEG data seems to be more focused, so that head misalignment among different sessions should be checked and eventually corrected if physiological signals are merged over time.
Reproducibility of these findings is currently under investigation by extending the sample for both the EEG and MEG measures.

Acknowledgments This project was partially supported by the DCMC Project of the Italian Space Agency. Aston University MEG Laboratory is supported by the Dr. Hadwen Trust. Support from the Aston University Visiting Professor Scholar Fund for Luigi Bianchi.

\section{References}

Allison BZ, Pineda JA (2006) Effects of SOA and flash pattern manipulations on ERPs, performance, and preference: implications for a BCI system. Int J Psychophysiol 59(2):127-140

Bianchi L, Babiloni F, Cincotti F, Salinari S, Marciani MG (2003) Introducing $\mathrm{BF}++$ : a $\mathrm{C}++$ framework for cognitive biofeedback systems design. Methods Info Med 42:104-110

Bianchi L, Quitadamo LR, Marciani MG, Maraviglia B, Abbafati M, Garreffa G (2007) How the NPX data format handles EEG data acquired simultaneously with FMRI. Magn Reson Imaging 25(6): 1011-1014

Donchin E, Spencer KM, Wijesinghe R (2000) The mental prosthesis: assessing the speed of a P300-based brain-computer interface. IEEE Trans Rehabil Eng 8(2):174-179

Krusienski DJ, Sellers EW, McFarland DJ, Vaughan TM, Wolpaw JR (2008) Toward enhanced P300 speller performance. J Neurosci Methods 167:15-21

Mellinger J, Schalk G, Braun C, Preissl H, Rosenstiel W, Bribaumer N, Kuebler A (2007) An MEG-based brain-computer interface (BCI). Neuroimage 36:581-593

Nijboer F, Sellers EW, Mellinger J, Jordan MA, Matuz T, Furdea A, Halder S, Mochty U, Krusienski DJ, Vaughan TM, Wolpaw JR, Birbaumer N, Kübler A (2008) A P300-based brain-computer interface for people with amyotrophic lateral sclerosis. Clin Neurophisiol 119(8):1909-1916

Sellers EW, Krusienski DJ, McFarland DJ, Vaughan TM, Wolpaw JR (2006) A P300 event-related potential brain-computer interface (BCI): the effects of matrix size and inter stimulus interval on performance. Biol Psychol 73(3):242-252 
Serby H, Yom-Tov E, Inbar GF (2005) An improved P300-based brain computer interface. IEEE Trans Neural Syst Rehabil Eng 13(1):88-98
Wolpaw JR, Birbaumer N, McFarland DJ, Pfurtscheller G, Vaughan TM (2002) brain-computer interfaces for communication and control. Clin Neurophysiol 113(6):767-791 